\title{
BMJ Open Prevalence of hospital-acquired infections (HAIs) and associated factors in Ethiopia: a systematic review and meta-analysis protocol
}

\author{
Birhan Gebresillassie Gebregiorgis (D , , Goitom Molalign Takele, ${ }^{2}$ \\ Kassahun Dires Ayenew, ${ }^{3}$ Yosef Eshetie Amare ${ }^{4}$
}

To cite: Gebregiorgis BG, Takele GM, Ayenew KD, et al. Prevalence of hospitalacquired infections (HAls) and associated factors in Ethiopia: a systematic review and metaanalysis protocol. BMJ Open 2020;10:e042111. doi:10.1136/ bmjopen-2020-042111

- Prepublication history for this paper is available online. To view these files, please visit the journal online (http://dx.doi. org/10.1136/bmjopen-2020042111).

Received 25 June 2020 Revised 25 November 2020 Accepted 01 December 2020

Check for updates

(C) Author(s) (or their employer(s)) 2020. Re-use permitted under CC BY-NC. No commercial re-use. See rights and permissions. Published by BMJ.

${ }^{1}$ Nursing, Debre Berhan University, Debre Berhan, Amhara, Ethiopia

${ }^{2}$ Nursing, Mekelle University, Mekelle, Tigray, Ethiopia

${ }^{3}$ Pharmacy, Debre Berhan University, Debre Berhan, Amhara, Ethiopia

${ }^{4}$ Biomedical Science, Debre Berhan University, Debre Berhan, Amhara, Ethiopia

Correspondence to Dr Birhan Gebresillassie Gebregiorgis;

birhan1475@gmail.com

\section{ABSTRACT}

Introduction Hospital-acquired infections (HAls) are public health problems of global concern and are notably prevalent in developing countries. The prevalence of $\mathrm{HAl}$ and its associated factors are not well described in the context of Ethiopia. Currently, the nationwide prevalence of HAl and its corresponding associated factors have not been formally reported in Ethiopia. This review will provide an estimate of the prevalence of HAl and its associated factors.

Methods Scholarly articles will be selected from the Embase, PubMed, Cochrane library, Hinary, Scopus, Web of Science and Google Scholar databases. Articles within the timeline of January 2000 to December 2020 will be included for review. Observational studies, randomised trials, surveys, surveillance reports, published and grey literature that reported the prevalence of HAl or factors associated with $\mathrm{HAl}$ reported as OR $(95 \% \mathrm{Cl})$ with no language restriction will be included in the analysis. Screening and selection of articles will be done using webbased Covidence software. The article's quality and risk of bias will be critically appraised using Johanna Briggs Institute quality appraisal checklist. Random effects model using the inverse variance method will be conducted to estimate the prevalence of HAl. To examine heterogeneity, the $Q$ statistics and $I^{2}$ statistics will be conducted. Publication bias will be investigated using Begg's correlation method and Egger's weighted regression test. All analyses will be performed with STATA (V.14) software. Ethics and dissemination Ethical approval is not required for meta-analysis reviews as participants are not included. The review will be submitted for publication in peer-reviewed journals.

\section{INTRODUCTION}

Hospital-acquired infections (HAIs)-also called nosocomial infections-are defined as infections acquired in a hospital by a patient who was admitted for a reason other than that infection. ${ }^{1}$ According to WHO, HAIs are important public health concerns leading to prolonged hospital stays, increase health system burden, negative impacts on patients and higher rates of mortality.
Strengths and limitations of this study

- No language restriction was imposed.

- The robustness of articles will be verified using welleffective quality appraisal tools.

- Absence of uniform diagnostic criteria for hospitalacquired infection.

HAIs occur worldwide regardless of levels of national development. ${ }^{2}$ Common HAIs include central line-associated bloodstream infections, catheter-associated infections, ventilator-associated pneumonia and surgical site infections. ${ }^{3}$

At any point in time, 10 out of every 100 hospitalised patients treated in developing countries will acquire at least one HAI. ${ }^{4}$ The 2017 Global Burden of Disease Study (GBD) ranked Ethiopia as having a Sociodemographic Index of approximately $0.334^{5}$ leaving it well within the parameters of a 'developing country' Its healthcare quality continues to be ranked among the lowest globally. ${ }^{6}$ While Ethiopia struggles with a wide variety of prevalent health concerns, infectious conditions such as respiratory infections, diarrhoeal diseases, HIV/AIDS and tuberculosis have continued to be its major health problems. ${ }^{78}$ In 2017 , these infectious diseases caused a highly significant portion of deaths in the country. ${ }^{8}$

A meta-analysis of studies in Ethiopia showed only half of healthcare workers practiced safe infection prevention practices. ${ }^{9}$ Though it varies by geographic region and from clinic to clinic, $15.3 \%-46.3 \%$ of healthcare workers in Ethiopia were not knowledgeable about infection prevention practices. ${ }^{10-12}$ Lack of knowledge among healthcare workers and poor prevention strategies in place may lead to a high risk of infection transmission in healthcare settings. 
Nationwide data on the prevalence of HAI and its risk factors is needed to develop and implement effective infection prevention and control strategies. However, in Ethiopia, the burden of HAI is not well described ${ }^{13-15}$ and data about risk factors of HAI is scarce.${ }^{16}$ An initial review of relevant databases provided no research or systematic review and meta-analysis that reported the nationwide prevalence of HAIs in Ethiopia. The proposed systematic review and meta-analysis seeks to estimate the prevalence and evaluate the factors associated with HAI among patients admitted to health facilities in Ethiopia.

\section{METHODS}

\section{Protocol preparation and results reporting}

This protocol is written based on the Preferred Reporting Items for Systematic Reviews and Meta-Analyses Protocols (PRISMA-P) 2009 guideline $^{17}$ (see online supplemental file 1) and Guidelines for Accurate and Transparent Health Estimates Reporting statement ${ }^{18}$ (see online supplemental file 2). The results will be reported following these guidelines. The PRISMA flow diagram will be used to demonstrate how articles were screened and selected for inclusion in the analysis (see online supplemental file $3)$.

\section{PECO search guide}

P: Patients who are admitted to a healthcare facility regardless of sex or age.

E: Exposure for HAI includes admission to a healthcare facility and the characteristics of patients present before and during admission.

C: Comparisons will be made across subgroups (eg, age, sex, admittance ward, surgical conditions)

O: hospital-acquired infections: For this review, HAIs are defined as infections occurring in a patient in a hospital or other healthcare facility in whom the infection was not present at the time of admission including but not limited to central line-associated bloodstream infections, catheter-associated infections, ventilator-associated pneumonia and surgical site infections.

\section{Data source and search strategy}

A primary search across all databases will be conducted to gather keywords, medical subject headings and free texts enclosed in titles and abstracts of similar articles. Subsequently, We will search for articles in Embase, PubMed, Cochrane library, Hinary, Scopus and Web of Science using search terms such as: "hospital-acquired infections", "HAI", "Healthcare-Associated Infections, "HCAI", "nosocomial infections", "risk factors", "Associated factors", "determinants" and "Ethiopia". The search string was constructed by following the recommendation presented by a recently published article. ${ }^{19}$ The search strategy has been prepared and can be accessed online (see online supplemental file 4). Google Scholar and BIOSIS previews will be used to search for unpublished works of literature.

\section{Eligibility criteria}

\section{Inclusion criteria}

- Cross-sectional, case-control and cohort studies, randomised trials, surveys and surveillance reports conducted in Ethiopia between January 2000 and December 2020 that reported the prevalence of HAI studies, reporting data on the associated factors of HAI reported as OR with $95 \%$ CIs or providing adequate data to produce these estimates published in any language will be included.

\section{Exclusion criteria}

- Case series, case reports and qualitative studies.

- Articles that can not be accessed even after contacting the author.

\section{Selection of studies}

Software-based screening and selection of studies will be conducted using Covidence. Two reviewers (BGG and GMT) will review and select the titles and abstracts based on the eligibility criteria. If a disagreement occurs among them, a third reviewer acting as a tie-breaker will elect to include or exclude the study. On inclusion, the full text of all selected articles will be imported to Covidence for further assessment of their appropriateness for quality appraisal and analysis. Bibliographies of selected articles will be reviewed and referenced studies will be considered for inclusion in the analysis if found to be suitable.

\section{Quality assessment and risk of bias}

The Johanna Briggs institution (JBI) quality appraisal checklists ${ }^{20}$ will be used to critically appraise each article's validity, value, relevance, risk of bias and trustworthiness of results. These checklists have been validated by the JBI scientific committee following an extensive review. All articles selected for inclusion in the analysis will undergo a rigorous appraisal by two reviewers (BGG and GMT) using JBI appraisal tools to analyse methodological qualities and to determine the extent to which a study has addressed the possibility of bias in its design, conduct and analysis. Articles that scored $\geq 50 \%$ of the quality assessment criteria will be considered as having a low risk of bias. Discrepancies between the reviewers will be resolved through either discussion or involvement of a third reviewer.

\section{Data extraction}

Articles included for final analysis will then be processed through the data extraction procedures with the help of Covidence web-based software after a customised data extraction table is created. The extraction table will be used to extract data about characteristics of the study (first author, study design, year of publication, location, hospital ward, sampling method, sample size and response rate), characteristics of participants (age group, sex, comorbidities, isolated infectious agent on culture), the prevalence of HAI, OR for associated factors or frequency of outcome among exposed and non-exposed. If a complete set of data cannot be extracted from the 
article, the corresponding author will be contacted for additional information.

\section{Data synthesis and analysis}

We will perform a random-effects model to determine the pooled estimate of the prevalence of HAI. First, the individual study weights will be obtained by the binomial equation for variance based on the inverse variance method. Afterwards, the pooled prevalence estimate of HAI will be determined with SE and CI. If the proportions get closer to the limits of 0.1 , the double arcsine transformation method ${ }^{21}$ will be employed to address both the problems of confidence limits outside the 0.1 range and that of variance instability. Forest plots will be used to represent the individual and pooled estimates of the prevalence. For associated factors, we will pool the log-transformed OR and the SE of the log OR. We will include associated factors that were investigated in at least two studies given the factor is defined similarly across all studies. Effect sizes will be conveyed in terms of prevalence and ORs with 95\% CI.

Heterogeneity among the eligible studies will be assessed with the $Q$ statistics and $\mathrm{I}^{2}$ statistics. ${ }^{22}{ }^{23}$ To unravel methodological sources of heterogeneity, metaregression analysis will be conducted. If statistical analysis is impossible due to substantial heterogeneity, the findings will be narrated using tables and figures. Publication and small studies bias will be assessed with Begg's rank correlation methods ${ }^{24}$ and Egger's weighted regression test. ${ }^{25}$ We will deal with publication bias using trim and fill analysis. ${ }^{26}$ The statistical analysis will be performed by STATA (V.14).

\section{Sensitivity analysis}

Sensitivity analysis will be conducted to see if the decisions made during the review process affected the overall result and conclusion. Taking the study's quality or sample size into consideration, we will perform the analysis by either adding or removing studies. The robustness of the review process will then be examined by the effect seen on the outcome estimate.

\section{Subgroup analysis}

If adequate data can be extracted from the eligible studies, a subgroup analysis will be conducted based on the different types of participants (age, sex, presence or absence of surgical procedures, admittance ward), regions where studies are conducted, study type and sample size.

\section{Public and patient involvement}

Including public and patient involvement (PPI) statements aligns closely with BMJ Open's values of transparency and inclusiveness. We hope that including PPI statements in all articles is the first step of many for $B M J$ Open in encouraging patient involvement.

\section{Ethics and dissemination}

Ethical approval is not necessary as this review will not involve patients or individuals as study participants. We will submit the review report to peer-reviewed journals for publication.

\section{DISCUSSION}

Infectious diseases are of major concern to Ethiopia's healthcare system. Healthcare facilities can inadvertently facilitate the transmission of infections between patients and healthcare workers increasing the risk of morbidity and mortality unless proper evidence-based and effective infection prevention strategies are implemented. This review will provide the pooled estimate of the prevalence of HAI and pooled OR for risk factors. This is intended to promote the implementation of more evidence-based policies and practice towards controlling the occurrence of HAI in healthcare facilities.

\section{Potential methodological amendments}

If it is mandatory to make methodological amendments, the reviewers will provide logical justification and the details of amendments on the publication of the review.

Correction notice This article has been corrected since it first published. The provenance and peer review statement has been included.

Twitter Birhan Gebresillassie Gebregiorgis @BGebreselase

Contributors BGG proposed the review question and designed a search strategy. BGG, GMT, KDA and YEA prepared the protocol. BGG and GMT planned article selection and aimed to perform the analysis. All authors have critically appraised the review protocol and provided their constructive comments.

Funding The authors have not declared a specific grant for this research from any funding agency in the public, commercial or not-for-profit sectors.

Competing interests None declared.

Patient consent for publication Not required.

Provenance and peer review Not commissioned; externally peer reviewed.

Supplemental material This content has been supplied by the author(s). It has not been vetted by BMJ Publishing Group Limited (BMJ) and may not have been peer-reviewed. Any opinions or recommendations discussed are solely those of the author(s) and are not endorsed by BMJ. BMJ disclaims all liability and responsibility arising from any reliance placed on the content. Where the content includes any translated material, BMJ does not warrant the accuracy and reliability of the translations (including but not limited to local regulations, clinical guidelines, terminology, drug names and drug dosages), and is not responsible for any error and/or omissions arising from translation and adaptation or otherwise.

Open access This is an open access article distributed in accordance with the Creative Commons Attribution Non Commercial (CC BY-NC 4.0) license, which permits others to distribute, remix, adapt, build upon this work non-commercially, and license their derivative works on different terms, provided the original work is properly cited, appropriate credit is given, any changes made indicated, and the use is non-commercial. See: http://creativecommons.org/licenses/by-nc/4.0/.

ORCID iD

Birhan Gebresillassie Gebregiorgis http://orcid.org/0000-0002-6886-5145

\section{REFERENCES}

1 World Health O. Prévention des infections nosocomiales : guide pratique. 2nd ed. Genève: Organisation mondiale de la Santé, 2008.

2 World Health O. Core components of infection prevention and control programs in health care 2011. Available: https://www.who.int/csr/ resources/publications/AM_core_components_IPC/en/

3 Prevention CfDCa. Health care associated infections 2014. Available: https://www.cdc.gov/hai/infectiontypes.html

4 Organization WH. Health care-associated infections fact sheet, 2016. 
5 Network GBoDC. Global burden of disease study 2017 (GBD 2017) socio-demographic index (SDI) 1950-2017. Seattle, United States: Institute for Health Metrics and Evaluation (IHME), 2018.

6 GBD 2016 Healthcare Access and Quality Collaborators. Measuring performance on the healthcare access and quality index for 195 countries and territories and selected subnational locations: a systematic analysis from the global burden of disease study 2016 . Lancet 2018;391:2236-71.

7 Crummey DE, Mehretu A, Marcus HG. Ethiopia: Encyclopaedia Britannica, 2019. Available: https://www.britannica.com/place/ Ethiopia

8 (IHME) IfHMaE. Ethiopia profile Seattle, WA IHME, University of Washington, 2018. Available: http://www.healthdata.org/Ethiopia

9 Sahiledengle B, Tekalegn Y, Woldeyohannes D. The critical role of infection prevention overlooked in Ethiopia, only one-half of healthcare workers had safe practice: a systematic review and metaanalysis. medRxiv 2020;2020:05.07.20094672.

10 Yazie TD, Sharew GB, Abebe W. Knowledge, attitude, and practice of healthcare professionals regarding infection prevention at Gondar university referral Hospital, Northwest Ethiopia: a cross-sectional study. BMC Res Notes 2019;12:563.

11 Desta M, Ayenew T, Sitotaw N, et al. Knowledge, practice and associated factors of infection prevention among healthcare workers in Debre Markos Referral Hospital, Northwest Ethiopia. BMC Health Serv Res 2018;18:465.

12 Geberemariyam BS, Donka GM, Wordofa B. Assessment of knowledge and practices of healthcare workers towards infection prevention and associated factors in healthcare facilities of West Arsi district, Southeast Ethiopia: a facility-based cross-sectional study. Arch Public Health 2018;76:69.

13 Ali S, Birhane M, Bekele S, et al. Healthcare associated infection and its risk factors among patients admitted to a tertiary hospital in Ethiopia: longitudinal study. Antimicrob Resist Infect Control 2018;7:2
14 Yallew WW, Kumie A, Yehuala FM. Point prevalence of hospitalacquired infections in two teaching hospitals of Amhara region in Ethiopia. Drug Healthc Patient Saf 2016;8:71-6.

15 Endalafer N, Gebre-Selassie S, Kotiso B. Nosocomial bacterial infections in a tertiary hospital in Ethiopia. $J$ Infect Prev 2011;12:38-43.

16 Tolera M, Marami D, Abate D, et al. Are invasive procedures and a longer hospital stay increasing the risk of healthcareassociated infections among the admitted patients at Hiwot Fana specialized university Hospital, eastern Ethiopia? Adv Prev Med 2020;2020:6875463:1-7.

17 Moher D, Liberati A, Tetzlaff J, et al. Preferred reporting items for systematic reviews and meta-analyses: the PRISMA statement. PLoS Med 2009;6:e1000097.

18 Stevens GA, Alkema L, Black RE, et al. Guidelines for accurate and transparent health estimates reporting: the gather statement. Lancet 2016;388:e19-23.

19 Bramer WM, de Jonge GB, Rethlefsen ML, et al. A systematic approach to searching: an efficient and complete method to develop literature searches. J Med Libr Assoc 2018;106:531-41.

20 Solci M, Zaci M, Choi TEllan A, Zohi M, eds. Joanna Briggs Institute Reviewer's Manual. The Joanna Briggs Institute, 2017.

21 Barendregt JJ, Doi SA, Lee YY, et al. Meta-analysis of prevalence. J Epidemiol Community Health 2013;67:974-8.

22 Higgins JPT, Thompson SG. Quantifying heterogeneity in a metaanalysis. Stat Med 2002;21:1539-58.

23 Higgins JPT, Thompson SG, Deeks JJ, et al. Measuring inconsistency in meta-analyses. BMJ 2003;327:557-60.

24 Begg CB, Mazumdar M. Operating characteristics of a RANK correlation test for publication bias. Biometrics 1994;50:1088-101.

25 Egger M, Davey Smith G, Schneider M, et al. Bias in meta-analysis detected by a simple, graphical test. BMJ 1997;315:629-34.

26 Shi L, Lin L. The trim-and-fill method for publication bias: practical guidelines and recommendations based on a large database of meta-analyses. Medicine 2019;98:e15987-e. 